\title{
Treatment of onychomycosis with dilute topical povidone- iodine in a dimethylsulfoxide solvent system
}

\author{
Capriotti K ${ }^{1,2 *}$, Stewart, KP ${ }^{2,3}$, Pelletier JP${ }^{2,3}$ and Capriotti $\mathrm{J}^{2,3}$ \\ ${ }^{1}$ Dermatologist, Bryn Mawr Skin and Cancer Institute, Rosemont, Philadelphia, USA \\ ${ }^{2}$ Veloce BioPharma LLC, Fort Lauderdale, Florida, USA \\ ${ }^{3}$ Ophthalmology Consultants, PC Christiansted, VI, USA
}

\begin{abstract}
Introduction: Novel solutions of dilute povidone-iodine and dimethylsulfoxide have been employed in our practice for the treatment of onychomycosis. A retrospective review of our clinical experience with this regimen was undertaken to evaluate the tolerability and efficacy of this therapy and to determine if further prospective study may be beneficial.

Methods: An IRB-approved retrospective chart review of all patients employing this therapy in our practice for at least 24 weeks was completed. Clinical examination findings and microbiological culture results were analyzed for all patients before and after completion of 24 weeks of therapy.

Results: A total of thirteen patients were identified and all thirteen were included in this report. None discontinued use due to intolerance. There were no reported adverse advents. The Onychomycosis Severity Index was used to assess both initial involvement and improvement at the 24-week time point. Pre-treatment fungal cultures were positive in all thirteen patients. Post-treatment fungal cultures were positive in five patients.

Conclusion: In this retrospective review, onychomycosis patients topically treated with dilute povidone-iodine solutions have experienced both subjective and objective improvement. Toxicity of the dilute topical povidone-iodine regimen was not observed or reported. Further prospective, controlled studies of aqueous topical povidone-iodine solutions for onychomycosis and paronychia are warranted.
\end{abstract}

\section{Introduction}

Onychomycosis is one of the most common nails disorders observed by dermatologists, primary care providers and podiatrists. It is a condition in which a fungal infection involving the nail plate and nailbed causes disfigured and discolored nails with frequent disruption of the normal nail bed/nail plate architecture. Both incidence and prevalence are on the rise, with an estimated 35 million Americans affected [1-3]. All infections are initiated with some sort of trauma to the nail plate, nail bed, proximal/lateral nail folds or hyponychium. This trauma allows the fungi to access the nail bed, where it slowly proliferates causing the stratum corneum of the nail bed to slowly become hyperkeratotic, often resulting in onycholysis. The infection also involves the nail folds and nail plate. The nail may become thickened, dystrophic, cracked, discolored and painful.

Certain conditions may predispose individuals to onychomycosis. Immunodeficiency, poor peripheral circulation and diabetes are frequent co-morbidities [4]. Likelihood of infection also increases with age as the rate of nail growth declines in older populations. Frequent trauma from athletics or grooming practices has also been associated with higher rates of persistent fungal infection of the feet and toe nails [5]. Males used to be more frequently affected than females, but with the increase in popularity of pedicures the incidence in females has risen to nearly equal levels.

Onychomycosis is most frequently caused by fungi of the dermatophyte family. Dermatophytes thrive in dark, moist, warm environments which explains why infection is seen more commonly in toenails than in fingernails, as feet are often occluded in shoes for lengthy periods of time. Definitive diagnosis is typically obtained via fungal culture of the nails performed in the office setting, which allows for speciation as well.

Current treatment options for onychomycosis are marginally successful at best. There are oral and topical prescription and topical non-prescription treatment options available, but none have proven to be consistently effective. The FDA has approved one prescription nail lacquer (ciclopirox; Penlac ${ }^{\mathbb{R}}$, Sanofi Aventis), which has a cure rate of less than $10 \%$ [6]. The FDA-approved oral treatments terbinfine (Novartis, Lamisi $^{\circledR}$, Basel, Switzerland) and itraconazole (Janssen, Sporonox ${ }^{\circledR}$, Titusville, NJ) have cure rates ranging from $30-40 \%$, though definition of "cure" is inconsistent among published studies [7,8]. Systemic therapies also have inherent risk of hepatotoxicity and hematologic toxicity [9]. Over the counter options have not demonstrated any higher success rates than prescription drugs. All therapeutic approaches require lengthy treatment periods (often greater than one year), high patient compliance and high cost. The largest barrier in effective treatment of onychomycosis is the inability of the anti-fungal agent to reach the true nidus of infection, the subungual space and nail

Correspondence to: Capriotti K, Bryn Mawr Skin and Cancer Institute, 919 Conestoga Road, Building 2, Suite 106, Rosemont, PA 19010, USA, Tel: 267456 7956; E-mail: karacapriotti@gmail.com

Received: November 06, 2016; Accepted: November 22, 2016; Published: November 25, 2016 
plate, due to difficulty of penetration. To date no systemic or topical therapy has emerged as a clearly superior choice for first-line therapy is these common, intractable infections. We have employed aqueous dilute povidone-iodine solutions in dimethylsulfoxide (DMSO) in an effort to eradicate the nidus of fungal infection with a novel therapeutic approach.

\section{Patients and methods}

IRB approval was obtained to perform a retrospective chart review of patients treated at the Bryn Mawr Skin and Cancer Institute with dilute solutions of $1 \%$ povidone-iodine $(\mathrm{w} / \mathrm{w})$ in aqueous dimethyl sulfoxide (DMSO) twice daily for at least 24 weeks. All patients were known to our practice and had been previously diagnosed with onychomycosis according to published practice guidelines [10]. All identified cases had positive pre-treatment fungal cultures (Table 1). Pre-treatment degree of severity was determined using the Onychomycosis Severity Index (OSI) [11]. The OSI score is obtained by multiplying the score for the area of involvement (range, $0-5$ ) by the score for the proximity of disease to the matrix (range, 1-5). Ten points are added for the presence of a longitudinal streak or a patch (dermatophytoma) or for greater than $2 \mathrm{~mm}$ of subungual hyperkeratosis. Mild onychomycosis corresponds to a score of 1 through 5; moderate, 6 through 15; and severe, 16 through 35 .

The treatment consisted of applying the 1\% PVP-I/DMSO solution twice daily to the nail folds, subungual space and nail plate itself. Patients were instructed to allow the solution to absorb and dry for one minute before dressing with socks and shoes.

The aqueous DMSO solvent was chosen due to its penetration enhancing ability and its well-described non-toxicity [12-14]. The PVP-I concentration was chosen based on reported antimicrobial efficacy along with the known pharmaceutical chemistry of povidoneiodine topical solutions $[15,16]$. Patients were typically evaluated at 24-week intervals as per office routine. Fungal cultures were again obtained at the follow-up visit and the OSI was re-evaluated.

\section{Results}

A total of thirteen patients were identified and included in this retrospective study. Patient demographic, clinical and mycological data are detailed below (Table 1). None of the patients discontinued use due to intolerance. There were no reported adverse reactions to topical application of PVP-I/DMSO solution. The severity of Onychomycosis was graded both pre-treatment and after 24 weeks of BID use using the OSI as being mild, moderate or severe with the corresponding numerical score. Pre-treatment and post-treatment culture results were determined by using $\mathrm{Mycosel}^{\mathbb{}}{ }^{\mathbb{C}}$ culture medium.

\section{Discussion}

Topical onychomycosis treatment strategies require lengthy courses of therapy (i.e. $>12$ months) in order to allow the infected tissues to grow out and be replaced by newly deposited healthy nails. Anti-fungal resistance to the treating agent frequently develops during these extended treatment periods required by the inherently slow growth rate of the infected nails. Anti-fungal resistance and poor topical response rates are further complicated by the inability of most topical agents to effectively penetrate the subungual and periungual infectious tissues. This leads to chronic re-infection, even during treatment, and contributes the extremely low success rates of most onychomycosis therapies [17]. In addition to the commonly seen onychomycosis, the more infrequent but related problem of paronychia may also benefit treatment with an aqueous, non-toxic, antiseptic topical agent that can penetrate the periungual space [18].

Povidone-iodine (PVP-I) is a well-known, non-toxic, commonly used topical antiseptic with no reported incidence of fungal resistance. The efficacy of low-concentration iodophor systems has been well described both in vitro and in vivo including both planktonic and biofilm-related infections [19]. This is the first known series combining low dose iodophors, in this case povidone-iodine, with an aqueous DMSO delivery system capable of penetrating the superficial skin structures. This enables efficient delivery of the active agent to the subungual and periungual spaces. It is observed that by treating these subungual and periungual infectious foci, the re-infection of the nail plate during the long therapeutic course is prevented allowing the newly deposited nail to grow out in a fungus-free environment. Our results in this case series address this point. All 13 patients demonstrated positive cultures prior to initiation of treatment. At the 24-week point of treatment, $8 / 13$ patients $(62 \%)$ had negative culture results. The 5 patients that had positive culture results had baseline severe or moderate infections according to the OSI scale, with 2 patients having dermatophytomas present. Current therapies have been unable to access the masses of fungal hyphae that comprise the dermatophytoma, rendering this subset of infection the most difficult to treat. Of note, one of the patients with negative fungal culture at the 24 -week time point also had a dermatophytoma present at the initiation of treatment. This

Table 1. Patient Demographic, Clinical and Mycological Data.

\begin{tabular}{|c|c|c|c|c|c|c|}
\hline \multirow[t]{2}{*}{ Patient } & \multirow[t]{2}{*}{ Age } & \multirow[t]{2}{*}{ Gender } & \multicolumn{2}{|c|}{ OSI category/score } & \multirow[t]{2}{*}{ Culture- Week 0} & \multirow[t]{2}{*}{ Culture- Week 12} \\
\hline & & & Week 0 & Week 24 & & \\
\hline 01 & 53 & $\mathrm{~F}$ & Severe $/ 30^{*}$ & Severe/26 & Positive** & Positive** \\
\hline 02 & 49 & $\mathrm{~F}$ & Moderate/6 & Mild/3 & Positive** & Negative \\
\hline 03 & 47 & $\mathrm{~F}$ & Moderate/15 & Moderate/13 & Positive** & Positive** \\
\hline 04 & 71 & $\mathrm{~F}$ & Severe/17 & Moderate/14 & Positive** & Positive** \\
\hline 05 & 62 & $\mathrm{~F}$ & Severe/17 & Severe/16 & Positive** & Negative \\
\hline 06 & 36 & $\mathrm{~F}$ & Moderate/7 & Moderate/6 & Positive*** & Negative \\
\hline 07 & 67 & $\mathrm{~F}$ & Severe $/ 20^{*}$ & Severe/18 & Positive** & Positive** \\
\hline 08 & 57 & M & Moderate/15 & Moderate/10 & Positive** & Negative \\
\hline 09 & 60 & $\mathrm{~F}$ & Mild/5 & Mild/3 & Positive** & Negative \\
\hline 10 & 65 & M & Severe/ $20^{*}$ & Severe/10 & Positive** & Positive** \\
\hline 11 & 67 & M & Severe/18* & Severe/16 & Positive** & Negative \\
\hline 12 & 33 & M & Mild/3 & Mild/2 & Positive*** & Negative \\
\hline 13 & 31 & M & Moderate/6 & Mild/5 & Positive** & Negative \\
\hline
\end{tabular}

*Indicates the presence of dermatophytoma (mass of fungal hyphae present within the nail represented by a thick yellow streak in the nail). **Culture positive for Trichophyton mentagrophytes. ***Culture positive for Trichophyton rubrum. 
nail sign has been major impedance to effective treatment in the past and suggests this novel combination has the potential ability to access the nidus of infection that any other therapy has failed to do. None of the patients demonstrated complete eradication of the infection according to the OSI scale, but this is not unexpected as onychomycosis infections often take 48 weeks or longer to demonstrate cure in both the clinical and mycologic setting.

Another surprising result is the speed in which patients noted improvement of their infected toenails. Numerous patients claimed embarrassment at the sight of their toes and refused to wear open toed shoes in seasonal climates. After 24 weeks of therapy many patients noted an improvement in color of the nail itself and all of the patients wished to continue treatment past the 24 weeks point, as they were pleased with the improvement noted. Though we did not design the current study to assess the treatment effect on Pseudomonas, we suspect efficacy in this common co-morbid infection as well. It is important to keep in mind that these subjective results may be biased, as only patients that feel socially stigmatized or uncomfortable by the condition seek medical treatment.

Observation in our single center of $1 \%$ PVPI solutions in DMSO has been encouraging when used to treat onychomycosis. Further prospective studies of this topical system are being pursued for onychomycosis and paronychia.

\section{References}

1. Elewski BE, Charif MA (1997) Prevalence of onychomycosis in patients attending a dermatology clinic in northeastern Ohio for other conditions. Arch Dermatol 133: 1172-1173. [Crossref]

2. Gill D, Marks R (1999) A review of the epidemiology of tinea unguium in the community. Australas J Dermatol 40: 6-13. [Crossref]

3. Ghannoum MA, Hajjeh RA, Scher R (2000) A large-scale North American study of fungal isolates from nails: the frequency of onychomycosis, fungal distribution, and antifungal susceptibility patterns. J Am Acad Dermatol 43: 641-648.

4. Faergemann J, Correia O, Nowicki R, Ro BI (2005) Genetic predisposition-understanding underlying mechanisms of onychomycosis. J Eur Acad Dermatol Venereol 19(Suppl) 1: 17-19.
5. Baran R (2011) The nail in the elderly. Clin Dermatol 29: 54-60. [Crossref]

6. Bridgewater NJ (2006) Penlac (ciclopirox olamine 8\%). Prescribing Information, Sanofi-aventis.

7. Warshaw EM, Fett DD, Bloomfield HE, Grill JP, Nelson DB, et al. (2005) Pulse versus continuous terbinafine for onychomycosis: a randomized, double-blind, controlled trial. $J$ Am Acad Dermatol 53: 578-584.

8. Gupta AK, Konnikov N, Lynde CW (2001) Single-blind, randomized, prospective study on terbinafine and itraconazole for treatment of dermatophyte toenail onychomycosis in the elderly. J Am Acad Dermatol 44: 479-484.

9. Gupta AK, Chwetzoff E, Del Rosso J, Baran R (2002) Hepatic safety of itraconazole. $J$ Cutan Med Surg 6: 210-213. [Crossref]

10. Garcia-Doval I, Cabo F, Monteagudo B (2010) Clinical diagnosis of toenai onychomycosis is possible in some patients: cross-sectional diagnostic study and development of a diagnostic rule. Br J Dermatol 163: 743-751.

11. Carney C, Tosti A, Daniel R, Scher R, Rich P, et al. (2011) A new classification system for grading the severity of onychomycosis: onychomycosis severity index. Arch Dermatol 147: 1277-1282.

12. Horita A, Weber LJ (1964) Skin penetrating property of drugs dissolved in dimethylsulfoxide (dmso) and other vehicles. Life Sci 3: 1389-1395. [Crossref]

13. Williams AC, Barry BW (2004) Penetration enhancers. Adv Drug Deliv Rev 56: 603618. [Crossref]

14. Capriotti K, Capriotti JA (2012) Dimethyl sulfoxide: history, chemistry, and clinical utility in dermatology. J Clin Aesthet Dermatol 5: 24-26. [Crossref]

15. Gottardi W (1985) The influence of the chemical behaviour of iodine on the germicidal action of disinfectant solutions containing iodine. J Hosp Infect 6 Suppl A: 1-11. [Crossref]

16. Berkelman RL, Holland BW, Anderson RL (1982) Increased bactericidal activity of dilute preparations of povidone-iodine solutions. J Clin Microbiol 15: 635-639. [Crossref]

17. Piraccini BM, Sisti A, Tosti A (2010) Long-term follow-up of toenail onychomycosis caused by dermatophytes after successful treatment with systemic antifungal agents. Jour Am Acad Dermatol 62: 411-414.

18. Capriotti K, Capriotti JA (2015) Chemotherapy-associated paronychia treated with a dilute povidone-iodine/dimethylsulfoxide preparation. Clin Cosmetic Investig Dermatol 21: 489-491.

19. Capriotti K, Capriotti JA (2012) Topical iodophor preparations: chemistry, microbiology, and clinical utility. Dermatol Online J 18: 1. [Crossref]

Copyright: (C2016 Capriotti K. This is an open-access article distributed under the terms of the Creative Commons Attribution License, which permits unrestricted use, distribution, and reproduction in any medium, provided the original author and source are credited. 